\title{
Redshift distribution of gamma-ray bursts and star formation rate
}

\author{
A. Mészáros ${ }^{1,2}$, Z. Bagoly ${ }^{3}$, L. G. Balázs ${ }^{4}$, and I. Horváth ${ }^{5}$ \\ 1 Astronomical Institute of the Charles University, V Holešovičkách 2, 18000 Prague 8, Czech Republic \\ e-mail: meszaros@mbox.cesnet.cz \\ 2 Max Planck Institute for Astrophysics, Garching, Karl-Schwarzschild-Str. 1, Postfach 1317, 85741 Garching, Germany \\ 3 Laboratory for Information Technology, Eötvös University, 1117 Budapest, Pázmány P. s. 1/A, Hungary \\ e-mail: bagoly@ludens.elte.hu \\ 4 Konkoly Observatory, 1525 Budapest, POB 67, Hungary \\ e-mail: balazs@konkoly.hu \\ 5 Department of Physics, Bolyai Military University, 1456 Budapest, POB 12, Hungary \\ e-mail: horvath.istvan@zmne.hu \\ Received 10 July 2005 / Accepted 9 May 2006
}

ABSTRACT

\begin{abstract}
Aims. The redshift distribution of gamma-ray bursts collected in the BATSE Catalog is compared with the star formation rate. We aim to clarify the accordance between them. We also study the case of comoving number density of bursts monotonously increasing up to redshift $\simeq(6-20)$.

Methods. A method independent of the models of the gamma-ray bursts is used. The short and the long subgroups are studied separately.

Results. The redshift distribution of the long bursts may be proportional to the star formation rate. For the short bursts this can also happen, but the proportionality is less evident. For the long bursts the monotonously increasing scenario is also less probable but still can occur. For the short bursts this alternative seems to be excluded.
\end{abstract}

Key words. gamma rays: bursts - cosmology: miscellaneous

\section{Introduction}

The Burst and Transient Source Experiment (BATSE) instrument on the Compton Gamma Ray Observatory (Meegan et al. 2001) detected around 2700 gamma-ray bursts (GRBs). From this data set it follows that there are two different subgroups of GRBs, "short" and "long" ones (see, e.g., Balázs et al. 2003, and the references therein); the existence of further subgroups is not excluded (Horváth 1998, 2002; Hakkila et al. 2003; Borgonovo 2004).

The redshift ( $z$ ) distributions of the GRB subgroups are not satisfactorily known. For the long subgroup the redshifts are known for $\sim 40$ long GRBs from the afteglow measurements provided after 1997. On the other hand, for the short GRBs only one direct redshift is known (Greiner 2005). In the case of the further subgroups even the physical reality of these subgroups is in doubt (Hakkila et al. 2003). (For more details about these questions see, e.g., the surveys of Mészáros 2001, and Piran 2004.)

Here we concentrate on the question: Can the redshifts of GRBs be distributed in accordance with the distribution of the other objects arising in the star formation regions?

This question is discussed, e.g., by Porciani \& Madau (2001), and the question is answered in essence positively. Nevertheless, great care is still needed for three reasons. First, Porciani \& Madau (2001) note that - assuming different luminosity functions for GRBs - one can always be consistent with the observations. Thus, a study having minimal theoretical assumptions about the luminosity functions would be useful. Second, once one is in accordance with the star formation rate (hereafter SFR), an alternative distribution can also fit the data. For example, several papers (Mészáros \& Mészáros 1995, 1996;
Horváth et al. 1996; Reichart \& Mészáros 1997; Hogg \& Fruchter 1999; Norris et al. 2000; Schaefer et al. 2001; Lloyd-Ronning et al. 2002; Norris 2002; Bagoly et al. 2003; Lin et al. 2004) suggest that, alternatively, a permanent monotonous increase of the comoving number density of GRBs up to $z \simeq(10-20)$ is also possible. For example, Hogg \& Fruchter (1999) consider this scenario as an acceptable alternative. Hence, this alternative should also be studied. (Note that Hogg \& Fruchter (1999) consider also a third possibility: the density of GRBs is proportional to the total integrated stellar density. This scenario is found to be less probable, and therefore it will not be discussed here.) Third, the evidence has increased for the intrinsic difference between short and long GRBs (see Balázs et al. 2003, and references therein). But, it is not sure that both subgroups are in accordance with the SFR. Hence, a new comparison of GRB redshift distributions and star formation scenarios separately for the short and long GRBs is needed. In this paper a procedure will be provided for the two subgroups separately with minimal theoretical assumptions about both the SFR scenario and its alternative.

The paper is organized as follows. In Sect. 2 we discuss the method. Section 3 contains the theoretical calculations, compared with the observations from the BATSE Catalog. Section 4 verifies these theoretical calculations. Section 5 discusses the observational biases. Section 6 gives the comparison of theory with observations. In Sect. 7 the results are summarized.

\section{The method}

Balázs et al. (2003) studied the fluences and the durations of GRBs, collected in the BATSE Catalog (Meegan et al. 2001). 
For the fluence $F$ it is fulfilled

$F=\frac{(1+z) E_{\text {iso }}}{4 \pi d_{\mathrm{L}}(z)^{2}}=c(z) E_{\text {iso }}$,

where $E_{\text {iso }}$ is the total emitted energy of the object assuming isotropic emission, and where $d_{\mathrm{L}}(z)$ is the luminosity distance of the object at redshift $z$. Balázs et al. (2003) show that $\log F$ is distributed normally separately for the two subgroups, and if $c(z)$ and $E_{\text {iso }}$ are independent variables, then one has

$\sigma_{\log c(z)}^{2}+\sigma_{\log E_{\text {iso }}}^{2}=\sigma_{\log F}^{2}$,

where $\sigma$ is the dispersion for the given quantity denoted by the index. The independence seems to occur both for the short and long subgroups, respectively. The statistical fitting of $\log F$ allows - separately for the two subgroups - a normal distribution with a dispersion $\sigma_{\log F}$, and if $\sigma_{\log c(z)}$ and $\sigma_{\log E_{\text {iso }}}$ are assumed to be comparable, then the Cramer theorem says that both variables $\log E_{\text {iso }}$ and $\log c(z)$ should also be distributed normally again separately for the two subgroups. It is also possible that either $\sigma_{\log c(z)} \gg \sigma_{\log E_{\text {iso }}}$ or $\sigma_{\log c(z)} \ll \sigma_{\log E_{\text {iso }}}$ occurs. If this is the case, then nothing can be said about the distribution of the variable having a much smaller dispersions - it may be normal; however, the variable with dominating dispersion must be normal. In Balázs et al. (2003) it is argued that the condition $\sigma_{\log c(z)} \gg \sigma_{\log E_{\text {iso }}}$ is unlikely. Hence, only the normal distrubution for $\log E_{\text {iso }}$ is a reasonable conclusion. No detailed discussion about the distribution of $c(z)$, i.e. about the redshift distribution of GRBs, is provided by Balázs et al. (2003).

In this article we will concentrate on the redshift distributions of the two subgroups.

The key idea of this article is the following.

We assume that a given subclass of GRBs in the BATSE Catalog is distributed in accordance with the redshift distribution of the objects in the star formation regions. We then calculate the theoretically expected distribution of $\log c(z)$, and the theoretically expected dispersion $\sigma_{\log c(z) \text {,theor }}$. These theoretical calculations are general cosmological ones, and hold for any subsample. The theoretical dispersion can be compared with the observed $\sigma_{\log F}$. Because we have two different observational values of $\sigma_{\log F}$ for the two subgroups, we can well make this compare the two subgroups separately. We will use one $\sigma_{\log c(z) \text {,theor value }}$ twice - for the two subsamples of GRBs.

It is a necessary condition that $\sigma_{\log c(z) \text {,theor }}<\sigma_{\log F}$ be fulfilled. If this condition is not fulfilled, then the primary assumption must be rejected. For a given subgroup the condition may be fulfilled, but not fulfilled for the second one, because for the second subgroup the observed $\sigma_{\log F}$ values are smaller.

If $\sigma_{\log c(z) \text {,theor }} \ll \sigma_{\log F}$ holds, then the primary assumption is acceptable, because in this case $\log c(z)$ can have an arbitrary distribution. If $\sigma_{\log c(z)}$ and $\sigma_{\log E_{\text {iso }}}$ are comparable then both variables should be distributed normally. For $\log E_{\text {iso }}$ this is true (Balázs et al. 2003). But for $\log c(z)$ the requirement for normal distribution is artificial. Nevertheless, it can happen that the distribution "mimics" a normal distribution. This means that - mathematically - the functional behavior of the distribution of $\log c(z)$ is similar to a Gaussian curve. If this nearly Gaussian distribution of $\log c(z)$ occurs, then this function together with the normal distribution of $\log E_{\text {iso }}$ allows the acceptance of the primary assumption. If $\sigma_{\log E_{\text {iso }}} \ll \sigma_{\log c(z) \text {,theor }}<\sigma_{\log F}$ holds then the situation is similar; the primary assumption is acceptable, when the distribution of $\log c(z)$ mimics a Gaussian curve. This procedure does not need any assumption about the model of the GRB.
If one assumes an accordance of SFR and the occurence of GRBs, one has to specify a SFR for the calculation of the distribution of $c(z)$. The SFR is taken from the literature. Several papers (Madau 1995; Madau et al. 1996; Steidel et al. 1999; Porciani \& Madau 2001; Wilson et al. 2002; Tonry et al. 2003; Giavalisco et al. 2004a,b; Dahlen et al. 2004; Strolger et al. 2004) suggest the following behavior of the comoving number density for the star forming regions: 1 . At $z \simeq 1$ the density should be greater than at $z \simeq 0.1$ roughly by $\simeq 10$ times; 2 . at higher redshifts, up to $z \simeq 6$, the comoving number density should remain nearly constant, or there should be a weak decrease. Therefore, there should be four independent parameters in the description of the SFR: the two typical redshifts ( $z_{\text {break }}$, where the density is peaked; $z_{\max }$, up to which the rate is defined), and the two characteristic density ratios ([(density at the peak redshift $) /($ density at $z=0)$ ] and [(density at $\left.z_{\max }\right) /($ density at $\left.z=0)\right]$ ). There is also a fifth parameter, the density at $z=0$, but this value will always disappear from our calculations. The exact functional form of the SFR may be analytically expressed by different empirical functions. For example, in Strolger et al. (2004) a smooth four-parametric function is used. Nevertheless, other functional dependences may also be chosen that contain the four free parameters. We will take - in accordance with Wilson et al. (2002) - a power-law dependence of the form $\propto(1+z)^{D_{1}}$ between $z=0$ and $z_{\text {break }}$, and then $\propto(1+z)^{D_{2}}$ between $z_{\text {break }}$ and $z_{\max }$. Reasonable values of $D_{1}$ are roughly between 2 and 4 , and for $D_{2}$ between -1 and 0 . The reasonable values of parameters $z_{\text {break }}$ are around 1.0-1.5 and $z_{\max }$ around 4-6. These values define the range of parameters in the theoretical calculations for $\sigma_{\log c(z), \text { theor. }}$ Of course, all reasonable cosmological models should also be considered.

No "identical" number density is needed because the normalization constant - the fifth parameter - vanishes. Thus, under the "accordance", we simply mean a "proportionality".

We will also discuss the alternative scenario. For this alternative we take in the theoretical calculations a monotonous growth of the comoving number density of GRBs up to very high redshifts. Mathematically, this is obtained from the SFR density, if in it $z_{\max }=z_{\text {break }}$ is taken. Then we have a $\propto(1+z)^{D_{1}}$ behavior between $z=0$ and $z=z_{\max }$, where $z_{\max } \simeq$ (5-20). The parameter $D_{2}$ does not exist in this case. Thus, we have only two free parameters: $z_{\max }=z_{\text {break }}$ and $D_{1}$. Having one single theoretical value, this value should be compared twice with the observed $\sigma_{\log F}$ from the Balázs et al. (2003) values separately for the two suubgroups.

Let the real physical density of GRBs at the redshift $z$ be given by $n(z)$. Its unit is $\mathrm{Mpc}^{-1} \mathrm{yr}^{-1}$. Then the number of GRBs, being at the infinitesimal redshift interval $[z,(z+\mathrm{d} z)]$ and observed by an observer at $z=0$, is given by

$$
\begin{aligned}
& N(z) \mathrm{d} z= \\
& \quad n(z) \frac{4 \pi d_{\mathrm{PM}}^{2}\left(z, H_{0}, \Omega_{\mathrm{M}}, \Omega_{\Lambda}\right)}{(1+z)^{3}}\left(\frac{-c \mathrm{~d} t\left(z, H_{0}, \Omega_{\mathrm{M}}, \Omega_{\Lambda}\right)}{\mathrm{d} z}\right) \mathrm{d} z,
\end{aligned}
$$

where $c$ is the velocity of light, $H_{0}$ is the Hubble constant, $\Omega_{M}$ is the ratio of the density of matter to the critical density, $\Omega_{\Lambda}$ is the dimensionless cosmological constant. The function $\mathrm{d} t\left(z, H_{0}, \Omega_{\mathrm{M}}, \Omega_{\Lambda}\right) / \mathrm{d} z$ is dependent on $z$ and on the three cosmological parameters $\left(H_{0}, \Omega_{\mathrm{M}}, \Omega_{\Lambda}\right)$. The distance $d_{\mathrm{PM}}\left(z, H_{0}, \Omega_{\mathrm{M}}, \Omega_{\Lambda}\right)=\left(c / H_{0}\right) Q\left(z, \Omega_{\mathrm{M}}, \Omega_{\Lambda}\right)=d_{\mathrm{L}}(z) /(1+z)$ is the proper-motion distance. This formula is a standard cosmological one (Carroll et al. 1992).

Mathematically, $N(z=0)=0$, because $d_{\mathrm{PM}}(z=0)=0$. In addition, $d_{\mathrm{PM}}$ remains finite, if $z \rightarrow \infty$. Hence, to have $N(z)=0$ 
at the limit $z \rightarrow \infty$ one needs a slower than $n(z) \propto(1+z)^{5.5}$ increase for $z \rightarrow \infty$. In other words, if $n(z)$ remains finite, or runs to $\infty$ for $z \rightarrow \infty$ but slower than $\propto(1+z)^{5.5}$, then the zero limit at infinite redshift is ensured for $N(z)$. This is always the case here. This global behavior of $N(z)$ is independent of the cosmological parameters, and hence may "mimic" a Gaussian distribution both for the SFR and for the alternative scenario.

$n(z)$ is the real physical density of GRBs under the condition of isotropic emission. Nevertheless, this assumption is not a loss of generality. Assume that GRBs emit in the solid angle $\omega$ (in steradian), where $\omega<4 \pi$. Then the real physical density of GRBs is given by $n(z)(4 \pi / \omega)$, but the observed number of GRBs is lowered by the factor $\omega /(4 \pi)$, and hence all formulae about the observed quantities hold. Thus, our theoretical calculations are independent of the size of the beaming (Frail et al. 2001; Amati et al. 2003; Piran 2004; Ghirlanda et al. 2004).

Having $N(z)$, it is straightforward for any function $f(z)$ to define its mean $\overline{f\left(z_{1}, z_{2}\right)}$ and dispersion $\sigma_{f}^{2}$ in the interval $\left[z_{1}, z_{2}\right]$ $\left(z_{1}<z_{2}\right)$.

A widely accepted cosmological model is given by $\Omega_{\mathrm{M}}=$ $0.3, \Omega_{\Lambda}=0.7$, where $\Omega_{\mathrm{M}}+\Omega_{\Lambda}=1$ holds exactly (Tonry et al. 2003). Nevertheless, from the observational point of view, the case $\Omega_{\Lambda}=0$ with $\Omega_{\mathrm{M}} \simeq(0.2-0.4)$ cannot be excluded (see Mészáros 2002; Tonry et al. 2003, and references therein). Therefore, for maximal generality, we should discuss both cases. We will also discuss the case $\Omega_{M}=1$ with $\Omega_{\Lambda}=0$. This eventuality is rejected by observations, but it can serve as a verificator. In the case with $\Omega_{M}+\Omega_{\Lambda}=1$, if $\Omega_{\Lambda}$ is decreased from 0.7 to zero, one should obtain this limit. In the case with $\Omega_{\Lambda}=0$ the value $\Omega_{\mathrm{M}}$ can be increased from 0.2 to 1 , and one should again obtain this limit. Hence this case can be taken as the limit for verificator. We proceed similarly to Mészáros \& Mészáros (1995), Mészáros \& Mészáros (1996) and Horváth et al. (1996), where this simplest model was also discussed. The cosmological parameter $H_{0}$ need not be specified for our calculations.

\section{Expected values for $\sigma_{\log c(z), \text { theor }}$}

Now assume that $n(z)$ is in accordance with SFR. Then $n(z)$ may be described by

$n(z)=n_{0}(1+z)^{3}(1+z)^{D_{1}}, \quad 0 \leq z \leq z_{\text {break }}$,

$n(z)=n_{0}(1+z)^{3+D_{2}}\left(1+z_{\text {break }}\right)^{D_{1}-D_{2}}, \quad z_{\text {break }} \leq z \leq z_{\text {max }}$,

where $z_{\text {break }} \simeq(1.0-1.5), z_{\max } \simeq(4.0-6.0), D_{1} \simeq(2-4)$ and $D_{2} \simeq(-1-0)$, and where $n(z)=0$ for $z>z_{\max }$.

The extra $(1+z)^{3}$ factor in the definition of $n(z)$ is needed here because in our formulae $n(z)$ means the real physical proper density, not the comoving one. In the general case, one must calculate the integrals numerically. The final $\sigma_{\log c(z), \text { theor will de- }}$ pend on the four parameters: $z_{\text {break }}, z_{\max }, D_{1}$ and $D_{2}$. The value $n_{o}$ vanishes from the formulae, and need not be specified.

This behavior may easily be changed into the alternative scenario with a monotonous increase of the comoving density with $z_{\text {break }}=z_{\max }$ (i.e. no second part with the exponent $D_{2}$ ), and for $z_{\text {break }}=z_{\max }$ one can take any value between $z=5$ and $z=20$. Then the best value for $D_{1}$ seems to be 0.5 (Reichart \& Mészáros 1997) with a small scatter between $D_{1}=0$ and $D_{1}=1$.

Using the equations from the previous Section, one has for $\Omega_{\mathrm{M}}=1$ and $\Omega_{\Lambda}=0$

$c(z)=\left[4 \pi\left(c / H_{0}\right)^{2}(1+z) Q(z)^{2}\right]^{-1}=\left[(1+z) 4 \pi d_{\mathrm{PM}}^{2}\right]^{-1}$,
Table 1. The expected dispersion $\sigma_{\log c(z), \text { theor }}$ for the different parameters in $n(z)$.

\begin{tabular}{|c|c|c|c|c|c|c|}
\hline$\overline{D_{1}}$ & $\overline{D_{2}}$ & $z_{\text {break }}$ & $z_{\max }$ & $\begin{array}{l}\Omega_{\mathrm{M}}=1 \\
\Omega_{\Lambda}=0\end{array}$ & $\begin{array}{c}\Omega_{\mathrm{M}}=0.3 \\
\Omega_{\Lambda}=0\end{array}$ & $\begin{array}{l}\Omega_{\mathrm{M}}=0.3 \\
\Omega_{\Lambda}=0.7\end{array}$ \\
\hline & & & & $\sigma_{\log c(z), \text { theor. }}$ & $\sigma_{\log c(z) \text {,theor }}$ & $\sigma_{\log c(z), \text { theor. }}$ \\
\hline$\overline{2.5}$ & 0.0 & 1.0 & 4.0 & 0.51 & 0.35 & 0.39 \\
\hline 2.5 & 0.0 & 1.0 & 6.0 & 0.58 & 0.40 & 0.40 \\
\hline 2.5 & 0.0 & 1.5 & 4.0 & 0.46 & 0.31 & 0.36 \\
\hline 2.5 & 0.0 & 1.5 & 6.0 & 0.53 & 0.35 & 0.38 \\
\hline 2.5 & -1.0 & 1.0 & 4.0 & 0.51 & 0.35 & 0.42 \\
\hline 2.5 & -1.0 & 1.0 & 6.0 & 0.58 & 0.42 & 0.42 \\
\hline 2.5 & -1.0 & 1.5 & 4.0 & 0.46 & 0.32 & 0.38 \\
\hline 2.5 & -1.0 & 1.5 & 6.0 & 0.52 & 0.37 & 0.39 \\
\hline 3.0 & 0.0 & 1.0 & 4.0 & 0.49 & 0.34 & 0.39 \\
\hline 3.0 & 0.0 & 1.0 & 6.0 & 0.56 & 0.40 & 0.41 \\
\hline 3.0 & 0.0 & 1.5 & 4.0 & 0.44 & 0.30 & 0.35 \\
\hline 3.0 & 0.0 & 1.5 & 6.0 & 0.51 & 0.35 & 0.38 \\
\hline 3.0 & -1.0 & 1.0 & 4.0 & 0.51 & 0.36 & 0.42 \\
\hline 3.0 & -1.0 & 1.0 & 6.0 & 0.54 & 0.41 & 0.43 \\
\hline 3.0 & -1.0 & 1.5 & 4.0 & 0.44 & 0.31 & 0.37 \\
\hline 3.0 & -1.0 & 1.5 & 6.0 & 0.50 & 0.34 & 0.39 \\
\hline 3.5 & 0.0 & 1.0 & 4.0 & 0.48 & 0.33 & 0.39 \\
\hline 3.5 & 0.0 & 1.0 & 6.0 & 0.55 & 0.39 & 0.42 \\
\hline 3.5 & 0.0 & 1.5 & 4.0 & 0.42 & 0.28 & 0.34 \\
\hline 3.5 & 0.0 & 1.5 & 6.0 & 0.49 & 0.34 & 0.38 \\
\hline 3.5 & -1.0 & 1.0 & 4.0 & 0.48 & 0.35 & 0.42 \\
\hline 3.5 & -1.0 & 1.0 & 6.0 & 0.55 & 0.40 & 0.44 \\
\hline 3.5 & -1.0 & 1.5 & 4.0 & 0.41 & 0.29 & 0.35 \\
\hline 3.5 & -1.0 & 1.5 & 6.0 & 0.48 & 0.35 & 0.39 \\
\hline$\overline{0.0}$ & - & 6.0 & 6.0 & 0.48 & 0.53 & 0.31 \\
\hline 0.0 & - & 10.0 & 10.0 & 0.54 & 0.58 & 0.33 \\
\hline 0.0 & - & 20.0 & 20.0 & 0.59 & 0.63 & 0.34 \\
\hline 0.5 & - & 6.0 & 6.0 & 0.47 & 0.49 & 0.33 \\
\hline 0.5 & - & 10.0 & 10.0 & 0.49 & 0.54 & 0.36 \\
\hline 0.5 & - & 20.0 & 20.0 & 0.51 & 0.59 & 0.43 \\
\hline 1.0 & - & 6.0 & 6.0 & 0.47 & 0.45 & 0.34 \\
\hline 1.0 & - & 10.0 & 10.0 & 0.52 & 0.48 & 0.40 \\
\hline 1.0 & - & 20.0 & 20.0 & 0.56 & 0.52 & 0.50 \\
\hline
\end{tabular}

where $Q(z)=2\left(1-(1+z)^{-1 / 2}\right)$. Hence, calculating the dispersion of $\log c(z)$, it is enough to calculate the dispersion of $\log [(1+$ z) $\left.Q(z)^{2} / 4\right]=\log (\sqrt{1+z}-1)^{2}=2 \log (\sqrt{1+z}-1)$.

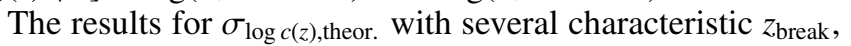
$z_{\max }, D_{1}$ and $D_{2}$ values are shown in Table 1.

$\sigma_{\log c(z) \text {,theor. }}$ does not depend strongly on the parameters $z_{\text {break }}, z_{\max }, D_{1}$ and $D_{2}$, because all values lead to the values (0.41-0.58).

The change given by the different $z_{\max }$ is negligible. In addition, if we change $z_{\max }$ to a larger interval (say, between $2 \leq z_{\max } \leq 10$; not written in the Table 1 ), the changes remain small. This is because $N(z)$ is decreasing at $z>2$.

Similarly, the changes given by the different values of $z_{\text {break }}$ are also small. If we change its value at a larger interval $(0.75 \leq$ $z_{\text {break }} \leq 2.0$; not written in Table 1$)$, the changes remain small. This is also expected from the behavior of $N(z)$, because it increases up to $z \simeq 1$, and then it decreases. The change of $z_{\text {break }}$ influences this behavior only weakly around the maximum of $N(z)$.

There is a clear tendency for $D_{1}$ and $D_{2}$ : increasing $D_{1}$ decreases the dispersion, and, similarly, decreasing $D_{2}$ also decreases $\sigma_{\log c(z) \text {,theor. }}$ Greater $D_{1}$ and/or smaller $D_{2}$ strengthens the "peakness" of $N(z)$, and thus decreases the dispersion because more objects are concentrated around $z_{\text {break }}$, where the mean should also be. Thus, one expects smaller dispersion.

We also calculated the values for the alternative scenario, when there is a monotonous $n(z) \propto(1+z)^{D_{1}}$ increasing up to 
very high redshift $z_{\max }$. Similar dispersions were obtained with a very weak dependence on $z_{\max }$ and $D_{1}$.

For the case $0<\Omega_{\mathrm{M}}<1, \Omega_{\Lambda}=0$ one may use $Q\left(z, \Omega_{\mathrm{M}}\right)=$ $2\left[2+\Omega_{\mathrm{M}}(z-1)-\left(2-\Omega_{\mathrm{M}}\right) \sqrt{\left.1+\Omega_{\mathrm{M}} z\right)}\right] /\left[\Omega_{\mathrm{M}}^{2}(1+z)\right]$. The key difference, compared to $\Omega_{M}=1$, follows from the smaller value involved here. We also probed several different $0<\Omega_{\mathrm{M}} \leq 1$ (not given in Table 1), and obtained a clear increase of $\sigma_{\log c(z), \text { theor }}$ with $\Omega_{\mathrm{M}}$. Otherwise the trends are identical to that of $\Omega_{\mathrm{M}}=1$. The alternative scenario shows similar trends with larger values.

For $\Omega_{\mathrm{M}}=0.3, \Omega_{\Lambda}=0.7$ we need to determine the values of $d_{\mathrm{PM}}$ numerically. The results are similar to that of $\Omega_{\mathrm{M}}=0.3$, $\Omega_{\Lambda}=0$. Thus, the values for $\Omega_{M}=0.3$ are similar in both cases, and depend weakly on the value of the cosmological constant. For the alternative scenario we obtain smaller values.

\section{K-correction at gamma range}

Before comparing the theoretical values of Table 1 with the fit of Balázs et al. (2003) we still discuss the values of Table 1 . This is needed because in the previous considerations the assumption of the independence of $c(z)$ and $E_{\text {iso }}$ was crucial. Because $c(z)$ is a function of $z$, this assumption is equivalent to the assumption that $E_{\text {iso }}$ does not depend on $z$ - a non-trivial assumption.

In our case $E_{\text {iso }}$ means the total emitted energy of the photons having energies $E_{1}(1+z) \leq E \leq E_{2}(1+z)$ under the assumption of isotropic emission. But this $E_{\text {iso }}$ can be different to that of the remitted total energy of the GRB in the interval $E_{1}(1+z) \leq E \leq$ $E_{2}(1+z)$ because we measured the fluence and the spectrum at the interval $E_{1} \leq E \leq E_{2}$, and from this measurement we made extrapolations about the emitted energy at the interval shifted by a factor $(1+z)$. Hence, $E_{\text {iso }}$ used in Eq. (1) may depend on $z$ even in the case when the real bolometric isotropic energies - emitted by GRBs - do not depend on $z$. In essence, we have the analogy of $\mathrm{K}$-correction in the gamma range.

This question is discussed by Bloom (2003) for GRBs, and here we will proceed similarly.

The case $E_{\text {iso }}=E_{\text {iso }}(z)$, where $E_{\text {iso }}$ is the quantity appearing in Eq. (1), may be reformulated as

$E_{\text {iso }}(z)=E_{\text {iso }}(0) q(z)$,

where $E_{\text {iso }}(0)$ is the emitted energy at $z=0$ between the photon energies $E_{1}$ and $E_{2}$, and $q(z)$ is some function of $z$ to be determined from the time integrated spectra of GRBs. Thus,

$F=\frac{(1+z) E_{\mathrm{iso}}(z)}{4 \pi d_{\mathrm{L}}(z)^{2}}=c(z) q(z) E_{\mathrm{iso}}(0)$.

This means that one should simply consider $q(z) c(z)$ instead of $c(z)$ itself, because then $q(z) c(z)$ and $E_{\text {iso }}(0)$ are independent variables. The effect of K-correction is included into the function $c(z)$, and - to be the most accurate - one has to estimate the theoretically expected values of $\sigma_{\log (q(z) c(z))}$, instead of the values $\sigma_{\log c(z)}$. We estimate from the spectra of GRBs how much the value of $q(z)$ can be changed between $z=0$ and, say, $z=20$. From its definition it follows that, mathematically, $q(z)$ is a dimensionless non-negative function of $z$, and $q(0)=1$.

Because we consider the whole emitted energy during the existence of a GRB, we should consider the time averaged spectra of GRBs. This can be represented by the semi-empirical Band spectrum (Band et al. 1993) taking the form

$S(E)=A_{1}(E / 100)^{\alpha} \exp \left(-E / E_{0}\right), \quad E \leq(\alpha-\beta) E_{0}$,

$S(E)=A_{2}(E / 100)^{\beta}, \quad E \geq(\alpha-\beta) E_{0}$, where $A_{2}$ takes the values ensuring the continuity at $E=(\alpha-$ $\beta) E_{0}$, and where $E$ and $E_{0}$ are in $\mathrm{keV}$. Then $S(E) \mathrm{d} E$ defines the number of received photons accross $\mathrm{cm}^{2}$ and having energies in the interval $[E,(E+\mathrm{d} E)]$. Several papers (Ryde \& Svensson 2000, 2002; Ryde \& Petrosian 2002) suggest that this form decribes the known GRB spectra; only the three parameters $\alpha, \beta, E_{0}$ should be varied. For long bursts the typical values are $\alpha=-1$, $\beta=-2$ and $E_{0}=150 \mathrm{keV}$ (Band et al. 1993). It is also known that this behavior can be extrapolated up to photon energies of tens of MeV (Tavani 1996). Remarkably, this behavior of spectra holds also for short bursts (Ghirlanda et al. 2004); the only difference is given by the fact that the $\propto E^{\alpha} \exp \left(-E / E_{0}\right)$ behavior holds in the discussed range leading to a much harder spectrum; hence $E_{0}$ should be much higher than hundreds of $\mathrm{keV}$, and the steeper slope with $\propto E^{-2}$ should not occur. The essentially different behavior of the short and long GRBs at the range $>300 \mathrm{keV}$ was recognized also by Balázs et al. (2004).

Taking $\alpha=-1$ and $\beta=-2$, and taking into account that for energies one should take $E S(E)$, one obtains an analytical formula for $q(z)$ in the form

$$
\begin{aligned}
q(z) & =\frac{\int_{(1+z) E_{1}}^{(1+z) E_{2}} S(E) E \mathrm{~d} E}{\int_{E_{1}}^{E_{2}} S(E) E \mathrm{~d} E} \\
& =\frac{\exp \left(-x_{1}(1+z)\right)-e^{-1}+e^{-1} \ln x_{2}+e^{-1} \ln (1+z)}{\exp \left(-x_{1}\right)-e^{-1}+e^{-1} \ln x_{2}},
\end{aligned}
$$

where $x_{1}=E_{1} / E_{0}$, and $x_{2}=E_{2} / E_{0}$. This formula holds for $E_{1}(1+z) \leq E_{0}$, if $E_{0} \leq E_{2}$. For higher $z \geq z_{0}$ one obtains $q(z)=$ const. $=q\left(z_{0}\right)$, where $1+z_{0}=E_{0} / E_{1}$. This formula can be used to estimate the behavior of $q(z)$ for the long bursts, because $E_{0} \leq E_{2}$ is fulfilled for long GRBs. On the other hand, for short burst one has $E_{0} \geq E_{2}$, and hence one may write

$$
\begin{aligned}
q(z) & =\frac{\int_{(1+z) E_{1}}^{(1+z) E_{2}} S(E) E \mathrm{~d} E}{\int_{E_{1}}^{E_{2}} S(E) E \mathrm{~d} E} \\
& =\frac{\exp \left(-x_{1}(1+z)\right)-\exp \left(-x_{2}(1+z)\right)}{\exp \left(-x_{1}\right)-\exp \left(-x_{2}\right)} \simeq \exp \left(-x_{1} z\right)
\end{aligned}
$$

because $\exp \left(-x_{2}\right) \ll \exp \left(-x_{1}\right)$, and $\exp \left(-x_{2}(1+z)\right) \ll$ $\exp \left(-x_{1}(1+z)\right)$ hold.

In the case of short GRBs one has $x_{1}<25 / 1000=0.025$, and hence up to $z=4, q(z)$ is between 1.0 and 0.9 ; i.e. the change is smaller than $10 \%$. For long bursts, substituting the different values of $z$ with $x_{1}=1 / 6=0.167, x_{2}=1000 / 150=6.67$ one may verify that up to $z=5$ the change of $q(z)$ is smaller than $16 \%$. Hence, assuming the accordance of $N(z)$ with SFR, the predicted dispersions in the previous Section are not influenced more than (10-16)\%. The effect should be smaller for short bursts. Because the parameters $\alpha, \beta, E_{0}$ may vary, more uncertainty should also be allowed, but even this variation in the parameters does not change the situation essentially, because the variation of parameters - on average - should cancel. Therefore, the estimation obtained for the typical values of $\alpha, \beta, E_{0}$ should hold. Hence a maximum $\simeq(10-16) \%$ uncertainty can be expected from this effect; it will be smaller for short GRBs. Bloom (2003) in an other discussion of this effect also allows a $\simeq 20 \%$ uncertainty. All this means that the values of Table 1 with $z_{\text {break }} \simeq 1$ can be used, but one should keep in mind that a $20 \%$ uncertainty may occur.

If one assumes $n(z) \propto(1+z)^{D_{1}}$ up to the very high redshifts, then for the long GRBs the situation will be even better, because for the very large redshifts (up to $z=20$ or so) 
the effects of K-correction should be even more negligible due to the behavior $S(E) \propto E^{-2}$ at the range of a few MeV keeping $q(z)$ constant. Contrary to this, for short GRBs this behavior need not be fulfilled, because for them there is no indication for $S(E) \propto E^{-2}$ around $1 \mathrm{MeV}$ from Ghirlanda et al. (2004). In other words, if the short bursts are at $z \simeq(5-15)$ in accordance with $n(z) \propto(1+z)^{D_{1}}$, then for them the K-correction will not be negligible, because $q(z)$ will differ from one (it should go to zero as $z$ increases).

\section{Biases}

In Sects. 2, 3 we have calculated the theoretically expected dispersions $\sigma_{\log c(z) \text {,theor }}$, and in the previous Section we argued that the values of Table 1 - with some care in the case of the alternative monotonous growth for short GRBs - can be used as the expected theoretical dispersions in the comparison with the observed $\sigma_{\log F}$. But it is well-known that the fluence itself is a biased quantity in the BATSE Catalog (see Balázs et al. 2003, for more details). Hence, the values of $E_{\text {iso }}$ can be biased. The theoretical values of $\sigma_{\log c(z), \text { theor }}$ from the previous sections can be used, but the observed values of $\sigma_{\log F}$ must be taken with care.

There are two different types of biases. The first type is given by the BATSE threshold leading to the fact that some faint GRBs are simply not detected. Then it is - in principle - possible that if they were also detected, $\sigma_{\log F}$ would be different. The second type of biasing follows from the fact that the fluences themselves are affected by systematic uncertainities, and if they were free of them, again it would be possible that $\sigma_{\log F}$ would be different.

These biases of the fluence are discussed in detail by Balázs et al. (2003). It is shown there that the first type may be overcame quite easily: it is enough to study only the bright GRBs. For example, if only the bursts with $P_{64}>1$ photon $/\left(\mathrm{cm}^{2} \mathrm{~s}\right)$ are taken ( $P_{64}$ is the peak-flux on the $64 \mathrm{~ms}$ trigger), then this truncated sample of bright GRBs should not be influenced by the threshold effects, and hence also by the first type of bias.

A problem can arise from the fact that this truncated bright sample might not represent the whole BATSE sample. If the value of $\sigma_{\log F}$ is used from the whole sample, then this value may not be precise due to the bias; if the value of $\sigma_{\log F}$ is used from the truncated bright sample, then this value is bias-free, but it may not represent the value of the whole sample.

This controversy in the general case would need a detailed study. Furtonately, in some special cases it is simply solvable. For example, this does not exist in the special case when the obtained values of $\sigma_{\log F}$ from both samples are identical.

For the whole sample of long GRBs, as it is given in Balázs et al. (2003), one has $\sigma_{\log F}=0.66$. For the truncated bright subsample with $P_{64}>1$ photon $/\left(\mathrm{cm}^{2} \mathrm{~s}\right)$ we obtained from a new calculation $\sigma_{\log F}=0.66$. Also for the short GRBs we obtained from the new calculations the same $\sigma_{\log F}=0.58$ value both for the full sample and for the truncated sample with $P_{64}>1$ photon $/\left(\mathrm{cm}^{2} \mathrm{~s}\right)$. Hence, the special case of indentical values is usable here. To check these identical values we calculated $\sigma_{\log F}$ for several limiting $P_{64}$. We obtained the behavior that $\sigma_{\log F}$ does not change for the truncated samples with $P_{64}>(0.2-2.5)$ photon/ $\left(\mathrm{cm}^{2} \mathrm{~s}\right)$; for even bigger $P_{64}$ there is a moderate decrease. This holds for both subgroups. Thus, the bias of the first type is unimportant in this article.

The second type of bias was shown to be unimportant by Balázs et al. (2003).

\section{Comparison of theoretical predictions with BATSE fluence dispersions}

For the long GRBs, if one assumes that they are distributed in accordance with SFR, it follows that $\sigma_{\log c(z) \text {,theor }}=(0.28-0.42)<$ 0.66 for $\Omega_{\mathrm{M}}=0.3$ independently of the value of the cosmological constant, and practically independent of the four parameters of SFR. Taking into account the uncertainty from the K-correction $(\simeq(16-20) \%)$ the condition $\sigma_{\log c(z), \text { theor }}<\sigma_{\log F}$ holds. Because, in addition, the distribution of $\log c(z)$ can mimic a normal distribution, the redshift distribution of long GRBs can be in accordance with the SFR.

For the short GRBs, the requirement of $\sigma_{\log c(z), \text { theor }} \simeq$ $(0.28-0.42)<0.58$ holds, but it is less obvious, because of the smaller values of the measured $\sigma_{\log F}$. The occurence of $\sigma_{\log c(z) \text {,theor }} \simeq \sigma_{\log F}$ cannot be excluded for $\Omega_{\mathrm{M}}=0.3$ (independently of the value of the cosmological constant). The relatively smaller K-correction $(\simeq 10 \%)$ does not change the situation. All this means that, contrary to the long GRBs, here the accordance with SFR is less certain.

If one considers the alternative scenarios, then $\sigma_{\log c(z) \text {,theor }} \simeq$ $\sigma_{\log F}$ cannot be excluded for $\Omega_{\mathrm{M}}=0.3$ for the long GRBs with zero cosmological constant (with non-zero cosmological constant the situation is better). On the other hand, if one considers $D_{1}=1$ and takes the whole BATSE sample with $\sigma_{\log F}=0.66$, then one can still arrive in an accordance. The situation was less satisfactory than for long ones assuming accordance with SFR.

For the short GRBs the validity of the alternative scenario is practically excluded because for any reasonable case $\sigma_{\log c(z) \text {,theor }} \simeq \sigma_{\log F} \simeq 0.58$ can occur. In addition, here also the K-correction may not be negligible.

\section{Conclusions}

Comparison of $\sigma_{\log c(z), \text { theor }}$ with the observational $\sigma_{\log F}$ gives the following results:

1. The comoving number density of the long GRBs in the BATSE Catalog may be proportional to SFR.

2. The comoving number density of the short GRBs in the BATSE Catalog can still be proportional to SFR, but the situation is less obvious than in the case of long GRBs.

3. For long GRBs in the BATSE Catalog the monotonous increase of the comoving number density in the form $\propto(1+z)^{D_{1}}$ up to $z \simeq(6-20)$ can still occur, but is less clear than the case when this density is proportional to SFR.

4. For the short GRBs in the BATSE Catalog, similar monotonous increase is excluded.

Acknowledgements. The authors thank to Giancarlo Ghirlanda, Peter Mészáros, Robert Mochkovitch, Elena Rossi, Hendrik Spruit, Gyula Szokoly, Martin Topinka, Gábor Tusnády and Roland Vavrek for the useful discussions. The valuable remarks of the anonymous referee are kindly acknowledged. A.M. thanks the Institute in Garching for warm hospitality. This study was supported by Hungarian OTKA grant No. T48870.

\section{References}

Amati, L., et al. 2003, A\&A, 390, 81

Bagoly, Z., Csabai, I., Mészáros, A., et al. 2003, A\&A, 398, 919

Balázs, L. G., Bagoly, Z., Horváth, I., Mészáros, A., \& Mészáros, P. 2003, A\&A, 401, 129

Balázs, L. G., Bagoly, Z., Horváth, I., \& Mészáros, A. 2004, Gamma-Ray Bursts in the Afterglow Era, in Proceedings of the Third Roma Symposium, ed. M. Feroci, F. Frontera, N. Masetti, \& L. Piro (ASP Publishing), 55

Band, D., Matteson, J., Ford, L., et al. 1993, ApJ, 413, 281

Bloom, J. S. 2003, AJ, 125, 2865

Borgonovo, L. 2004, A\&A, 418, 487 
Carroll, S. M., Press, W. H., \& Turner, E. L. 1992, ARA\&A, 30, 499 Dahlen, T., Strolger, L.-G., Riess, A. G., et al. 2004, ApJ, 613, 189 Frail, D. A., Kulkarni, S. R., Sari, R., et al. 2001, ApJ, 562, L55 Ghirlanda, G., Ghisellini, G., \& Celotti, A. 2004, A\&A, 422, L55 Giavalisco, M., Ferguson, H. C., Koekemoer, A. M., et al. 2004a, 600, L93 Giavalisco, M., Dickinson, M., Ferguson, H. C., et al. 2004b, 600, L103 Greiner, J. 2005, http: //www.mpe.mpg.de/ jcg/grbgen.html Hakkila, J., Giblin, T. W., Roiger, R. J., et al. 2003, ApJ, 582, 320

Hogg, D. W., \& Fruchter, A. S. 1999, ApJ, 520, 54

Horváth, I. 1998, ApJ, 508, 757

Horváth, I. 2002, A\&A, 392, 79

Horváth, I., Mészáros, P., \& Mészáros, A. 1996, ApJ, 470, 56

Lin, J. R., Zhang, S. N., \& Li, T. P. 2004, ApJ, 605, 579

Lloyd-Ronning, N. M., Fryer, C. L., \& Ramirez-Ruiz, E. 2002, ApJ, 574, 554

Madau, P. 1995, ApJ, 441, 18

Madau, P., Ferguson, H. C., Dickinson, M. E., et al. 1996, MNRAS, 283, 1388

Meegan, C. A., et al. 2001, Current BATSE Gamma-Ray Burst Catalog, http://gammaray.msfc.nasa.gov/batse/grb/catalog Mészáros, P. 2001, Science, 291, 79
Mészáros, A. 2002, ApJ, 580, 12

Mészáros, P., \& Mészáros, A. 1995, ApJ, 449, 9

Mészáros, A., \& Mészáros, P. 1996, ApJ, 466, 29

Norris, J. P. 2002, ApJ, 579, 386

Norris, J. P., Marani, G. F., \& Bonnell, J. T. 2000, ApJ, 534, 248

Piran, T. 2004, Rev. Mod. Phys., 76, 1143

Porciani, C., \& Madau, P. 2001, ApJ, 548, 522

Reichart, D. E., \& Mészáros, P. 1997, ApJ, 483, 597

Ryde, F., \& Svensson, R. 2000, ApJ, 529, L13

Ryde, F., \& Svensson, R. 2002, ApJ, 566, 210

Ryde, F., \& Petrosian, V. 2002, ApJ, 578, 290

Schaefer, B. E., Deng, M., \& Band, D. L. 2001, ApJ, 563, L123

Steidel, C. C., Adelberger, K. L., Giavalisco, M., Dickinson, M., \& Pettini, M. 1999, ApJ, 519, 1

Strolger, L.-G., Riess, A. G., Dahlen, T., et al. 2004, ApJ, 613, 200

Tavani, M. 1996, ApJ, 466, 768

Tonry, J. L., Schmidt, B. P., Barris, B., et al. 2003, ApJ, 594, 1

Wilson, G., Cowie, L. L., Barger, A. J., \& Burke, D. J. 2002, AJ, 124, 1258 